See discussions, stats, and author profiles for this publication at: https://www.researchgate.net/publication/3322843

\title{
Risk-sensitive maximum likelihood sequence estimation
}

Article in IEEE Transactions on Circuits and Systems I Fundamental Theory and Applications · October 1996

DOI: 10.1109/81.536754 · Source: IEEE Xplore

CITATIONS

8

3 authors, including:

Robert J. Elliott

University of Adelaide

593 PUBLICATIONS 9,830 CITATIONS

SEE PROFILE

Some of the authors of this publication are also working on these related projects:

Project Regime-switching models in finance View project

Project Hawkes Processes in Finance View project
READS

8

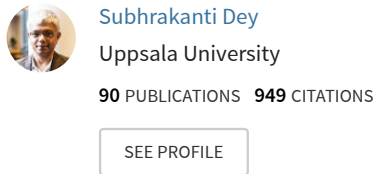


the original system. In fact, we have solved an optimal parameter selection problem. Instead of directly searching the poles and zeros of a reduced-order transfer function, we searched the Routh $\gamma$ parameters of the linear and quadratic factors of the numerator and denominator polynomials. This approach allows one to represent a reduced-order model in a unique form without prespecifying its pole-zero configuration. More importantly, it converts the stability constraints on the decision parameters into simple bounds. This feature allows one to utilize existing unconstrained gradient-based optimization techniques to find an optimal reduced-order model which has the same numbers of RHP poles and zeros of the original unstable and/or nonminimum-phase system. Finally, it should be mentioned that the effectiveness of a gradient-based optimization technique for model reduction depends closely on the initial guesses of the decision variables. Usually, the Routh $\gamma$ parameters associated with the dominant or unstable poles and zeros of the original system are good initial guesses which can converge to the true optimal values.

\section{REFERENCES}

[1] A. Bultheel and M. Van Barel, "Padé techniques for model reduction in linear system theory: A survey," J. Comput. Appl. Math., vol. 14, pp. $401-438,1986$.

[2] M. F. Hutton and B. Friedland, "Routh approximations for reducing order of linear time-invariant systems," IEEE Trans. Automat. Contr., vol. AC-20, pp. 329-337, Jun. 1975.

[3] A. S. Rao, S. S. Lamba, and S. V. Rao, "Routh-approximant timedomain reduced-order modeling for single-input, single-output systems," Proc. IEE, vol. 125, pp. 1059-1063, 1978.

[4] K. Glover, "All optimal Hankel-norm approximations of linear multivariable systems and their $L^{\infty}$-error bounds," Int. J. Contr., vol. 39, pp. $1115-1193,1984$.

[5] Y. S. Hung and K. Glover, "Optimal Hankel-norm approximation of stable systems with first-order stable weighting functions," Syst. Contr. Lett., vol. 7, pp. 165-172, 1986.

[6] B. C. Moore, "Principal component analysis in linear systems: controllability, observability and model reduction," IEEE Trans. Automat. Contr., vol. 26, pp. 17-31, Feb. 1981.

[7] V. Sreeram and P. Agathokils, "Model reduction using balanced realizations with improved low frequency behavior," Syst. Contr. Lett., vol. 12, no. 1, pp. 33-38, Jan. 1989.

[8] D. A. Wilson, "Optimal solution of model reduction problem," Proc. $I E E$, vol. 117, pp. 1161-1165, 1970.

[9] __ "Model reduction for multivariable systems," Int. J. Contr., vol. 20, pp. 57-64, 1974.

[10] L. R. Pujara and K. S. Rattan, "Model reduction of linear multivariable control systems via frequency matching," IEE Proc., vol. 131, Pt. D, pp. 243-247, 1984.

[11] _ "A frequency matching method for model reduction of digital control systems," Int. J. Contr., vol. 35, pp. 139-148, 1982.

[12] G. D. Howitt and R. Luus, "Model reduction by minimization of integral square error performance indices," J. Franklin Inst., vol. 327, no. 3, pp. 343-357, 1990.

[13] A. S. Rao, S. S. Lamba, and S. V. Rao, "On simplification of unstable systems using Routh approximation technique," IEEE Trans. Automat. Contr., vol. AC-23, pp. 943-944, 1978.

[14] _ _ "Reduced order models for unstable systems: Time domain approach," in Proc. Modeling and Simulation Conf., 1979, pp. 489-493.

[15] A. M. Guillaume and P. T. Kabamba, "Optimal finite horizon approximation of unstable linear systems," J. Guidance Contr., vol. 8, pp. $278-280,1985$.

[16] A. Zilouchian, "Balanced structures and model reduction of unstable systems," in Proc. IEEE Southeast Conf., vol. 2, 1991, pp. 1198-1201.

[17] C. Kenney and G. Hewer, "Necessary and sufficient conditions for balancing unstable systems," IEEE Trans. Automat. Contr., vol. AC-32, pp. $157-160$, Feb. 1987.

[18] C. P. Therapos, "Balancing transformation for unstable nonminimal linear systems," IEEE Trans. Automat. Contr., vol. 34, pp. 455-457, Apr. 1989.
[19] T. Y. Chiu, "Model reduction for unstable linear systems," Contr.-Theory Adv. Technol., vol. 9, no. 2, pp. 467-480, Jun. 1993.

[20] P. J. Parker and B. D. O. Anderson, "Unstable rational function approximation," Int. J. Contr., vol. 46, no. 5, pp. 1783-1801, 1987.

[21] D. G. Meyer, "A fractional approach to model reduction," in Proc. Amer. Contr. Conf., 1988, pp. 1041-1047.

[22] J. M. Santigao and M. Jamshidi, "On the extensions of the balanced approach for model reduction," Contr.-Theory Adv. Technol., vol. 2, pp. 207-226, 1986.

[23] C. S. Hsu and D. Hou, "Reducing unstable linear control systems via real Schur transformation," Electron. Lett., vol. 27, no. 11, pp. 984-986, May 1991.

[24] M. Vidyasagdar, "On undershoot and nonminimum phase zero," IEEE Trans. Automat. Contr., vol. AC-31, p. 440, 1986.

[25] M. Green, "Balanced stochastic realization," Linear Algebra Applicat., vol. 98, pp. 211-247, 1988.

[26] A. H. Bamani and R. W. Aldhaheri, "Combined Schur $/ L_{2}$-model reduction for nonminimal systems," Optimal Contr. Applicat. Methods, vol. 13, pp. 169-177, 1992.

[27] K. Zhou, "Relative/Multiplicative model reduction for unstable and nonminimal-phase systems," Automatica, vol 31, no. 8, pp. 1087-1098, 1995.

[28] S. Barnett and D. D. Siljak, "Routh's algorithm: a centennial survey," SIAM Rev., vol 19, no. 3, pp. 472-489, 1977.

[29] K. Walton and H. Gorecki, "On the evaluation of cost functionals, with particular emphasis on time-delay systems," IMA J. Math. Contr. Inform., vol. 1, no. 3, pp. 283-306, 1984.

[30] C. J. Demeure and C. T. Mullis, "The Euclid algorithm and the fast computation of cross-covariance and autocovariance sequences," IEEE Trans. Acoust, Speech, Signal Processing, vol. 37, pp. 545-552, 1989.

[31] D. J. Clements and B. D. O. Anderson, "Polynomial factorization via the Riccati equation," SIAM J. Appl. Math., vol. 31, no. 1, pp. 179-205, 1976.

[32] IMSL: User's Manual for IMSL MathLibrary, version 1.1, IMSL Inc., Houston, TX, 1989.

\section{Risk-Sensitive Maximum Likelihood Sequence Estimation}

\author{
R. J. Elliott, J. B. Moore, and S. Dey
}

Abstract-In this brief, we consider risk-sensitive Maximum Likelihood sequence estimation for hidden Markov models with finite-discrete states. An algorithm is proposed which is essentially a risk-sensitive variation of the Viterbi algorithm. Simulation studies show that the risk-sensitive algorithm is more robust to uncertainties in the transition probability matrix of the Markov chain. Similar estimation results are also obtained for continuous-range state models.

\section{INTRODUCTION}

The risk-sensitive optimization problem minimizes an exponential of a quadratic cost criterion to achieve an optimal estimation or control strategy. Risk-sensitive control problems have been studied in [1]-[4]. Recently a solution to the output feedback problem for linear and nonlinear discrete-time systems using information

Manuscript received May 24, 1995; revised November 13, 1995. This work was supported by the Cooperative Research Centre for Robust and Adaptive Systems, by the Australian Commonwealth Government under the Cooperative Research Centres Program. This paper was recommended by Associate Editor Y. Inouye.

R. J. Elliott is with the Department of Mathematics, University of Alberta, Edmonton, Alberta, Canada.

J. B. Moore and S. Dey are with the Department of Systems Engineering, The Australian National University, Canberra, Australia.

Publisher Item Identifier S 1057-7122(96)07183-8. 
state techniques has been proposed in [5] and [7]. Risk-sensitive estimation problems have received considerable attention recently [6], and [8]-[10]. The motivation is to obtain estimation and control strategies which are more robust than minimum variance estimation or control techniques, particularly in situations involving plant and noise uncertainties. This intended robustness is achieved by penalizing the higher order moments of the estimation error energy by the exponentiation operation. The index of the exponential is usually weighted by a risk-sensitive parameter (usually positive) which is a design parameter to be chosen based on the nature and extent of the uncertainty in the plant and/or noise models. Specifically, it has been mentioned in [9] that risk-sensitive filters for hidden Markov models (HMM) with finite-discrete states perform better than standard HMM filters in situations involving uncertainties in the noise statistics. Also, in the small noise limit, risk-sensitive problems have been shown to be closely related to worst-case estimation/control problems in a deterministic worst-case noise scenario connected to $H_{\infty}$ estimation/control [7] and [10].

The risk-sensitive filtering problem has been studied for general nonlinear signal models in [8] and similar results to [6] for linear signal models have been obtained as a special case. The same problem has been solved for hidden Markov models in [9] where recursive estimates have been defined and the optimizing state estimate is obtained as the minimizing argument of a function involving these recursive estimates. These recursions are linear and finite-dimensional as are the recursions for the "forward variable" or the true filtered estimate for the state of the hidden Markov chain obtained in [13]. A common feature of these recursions is that they involve a summation over all possible past states. It is well-known that a similar recursive structure with a maximization over the previous states rather than a summation appears in the Viterbi algorithm [13]. This was originally proposed for decoding convolutional codes in 1967. But, since then, it has found applications in Maximum Likelihood sequence estimation for a discrete-time finite-state Markov process observed in memoryless noise [14]. In this paper, we study a risksensitive variation of the Viterbi-type recursions with the motivation to achieve robust Maximum Likelihood sequence estimation. The authors would like to stress on the fact that although the risk-sensitive Maximum Likelihood sequence estimation strategy is an application of the optimal risk-sensitive estimation of HMM's, it is a robust but suboptimal variation of the Viterbi algorithm. Simulation studies show that the risk-sensitive algorithm yields substantially less number of decision errors in recovered data particularly when there is an uncertainty in the transition probability matrix of the Markov chain. This could be very useful in communication systems involving fast time-varying channels or military communication systems. Also, in the small noise limit, this risk-sensitive algorithm can be interpreted in terms of a deterministic worst-case measurement noise estimation strategy given from a differential game, which is closely related to $H_{\infty}$ estimation theory. This is quite a nice result in its own right. Details are not given this paper but the analysis follows closely that of [10]. Signal models considered in this paper are discrete-time and derivations involve change of probability measure techniques (developed in [12] and used in [7]-[9]).

In Section II, we describe the signal model and give brief details of the change of probability measure techniques in Section III. Section IV presents the results regarding recursive estimation of products of general real functions of the state given observations. In Section V, we study Viterbi-type recursions for estimating products of a certain class of such functions related to risk-sensitive estimation problems. These recursions along with a subsequent optimization yield an algorithm for what we call as the risk-sensitive Maximum Likelihood sequence estimation (RSMLSE). Section VI presents sim- ilar results for continuous-range state models. In Section VII simulation studies demonstrate robustness of the RSMLSE, and in Section VIII, we state some concluding remarks.

\section{SIGNAL MODEL}

Consider a probability space $(\Omega, \mathcal{F}, P)$. Let $X_{k}$ be a discrete-time homogeneous, first-order Markov process belonging to a finitediscrete set in this probability space. Define $\mathcal{E} \triangleq\left\{e_{1}, e_{2}, \cdots, e_{N}\right\}$ where $e_{i}=(0, \cdots, 0,1,0, \cdots, 0)^{\prime} \in \mathbb{R}^{N}$ with 1 in the $i$ th position. Without loss of generality, we can assume that $X_{k} \in \mathcal{E}$ [15]. Define $\mathcal{F}_{k}^{0}=\sigma\left\{X_{0}, \cdots, X_{k}\right\}$ and the corresponding complete filtration as $\left\{\mathcal{F}_{k}\right\}$. Also, there is an observation sequence $\left\{y_{k}\right\}$, $k \in \mathbb{N}$ which is a function of the Markov chain $X_{k}$ hidden in noise. The complete state-space description is then defined by

$$
\begin{aligned}
X_{k+1} & =A X_{k}+W_{k+1} \\
y_{k} & =C\left(X_{k}\right)+v_{k}
\end{aligned}
$$

where $W_{k}, k \in \mathbb{N}$ is a sequence of $\mathcal{F}_{k}$-martingale increments and hence $E\left[W_{k+1} \mid \mathcal{F}_{k}\right]=0$. Also, $y_{k} \in \mathbb{R}^{p}$ and $v_{k} \in \mathbb{R}^{p}, k \in \mathbb{N}$ is the measurement noise which is i.i.d with a strictly positive density function $\phi$. Here, $A$ is the transition probability matrix of the Markov chain $X_{k}$ where, $a_{i j}=P\left(X_{k+1}=e_{i} \mid X_{k}=e_{j}\right)$. Of course, $a_{i j}>0, \forall i, j, i, j \in\{1,2, \cdots, N\}$ and $\sum_{i} a_{i j}=1, \forall j \in$ $\{1,2, \cdots, N\}$.

Remark 11.1: Details about this formulation of the Markov process can be found in [12]. Also, the idempotent property of $X_{k}$ allows us to express nonlinear functions of $X_{k}$ as essentially linear functions and thus many derivations become quite simple (see [12] for examples).

Define $\mathcal{G}_{k}^{0}=\sigma\left\{X_{0}, X_{1}, \cdots, X_{k}, y_{0}, y_{1}, \cdots, y_{k-1}\right\}$ and $\mathcal{Y}_{k}^{0}=$ $\sigma\left\{y_{0}, y_{1}, \cdots, y_{k}\right\}$ and $\left\{\mathcal{G}_{k}\right\}$ and $\left\{\mathcal{Y}_{k}\right\}$ are the corresponding complete filtrations.

\section{Change of Probability Measure}

In this section, we use the change of probability measure technique which is based on a discrete-time version of Girsanov's theorem [11], Fubini's theorem, and Kolmogorov's extension theorem. A new probability measure is defined where the observations are independent and the estimation problem is solved in this new measure. The results can be interpreted as those in the old measure (i.e., where the real model is defined) as long as the Radon-Nikodym derivative of the old measure with respect to the new measure is restricted to satisfy a certain condition. More details can be found in [12].

Consider a new probability measure $\bar{P}$ under which the $y_{k}, k \in \mathbb{N}$ are i.i.d with density $\phi$. Define

$$
\begin{aligned}
& \bar{\lambda}_{k}=\frac{\phi\left[y_{k}-C\left(X_{k}\right)\right]}{\phi\left(y_{k}\right)} \\
& \bar{\Lambda}_{k}=\prod_{l=0}^{k} \bar{\lambda}_{l} .
\end{aligned}
$$

If we set the Radon-Nikodym derivative $d P /\left.d \bar{P}\right|_{\mathcal{G}_{k}}=\bar{\Lambda}_{k}$, then under $P$, the random variables $v_{k}, k \in \mathbb{N}$ are i.i.d with density functions $\phi$, where $v_{k}=y_{k}-C\left(X_{k}\right)$ (for proof, see [12]).

Remark III.1: Note that instead of continuous observations in the signal model (1), one can consider finite-discrete observations. In that case, the definition of $\bar{\lambda}_{k}$ will be different. Details can be found in [12].

Now, we use a version of Bayes' Theorem to obtain the following result which will be used often subsequently

$$
E\left[\Phi_{k} \mid \mathcal{Y}_{k}\right]=\frac{\bar{E}\left[\bar{\Lambda}_{k} \Phi_{k} \mid \mathcal{Y}_{k}\right]}{\bar{E}\left[\bar{\Lambda}_{k} \mid \mathcal{Y}_{k}\right]}
$$

where $\Phi_{k}$ is any $\mathcal{G}$-adapted sequence. 


\section{Recursive Estimation OF Products}

In this section, we present the results regarding recursive estimation of products of real functions of the state given the observations. We define an unnormalized measure which can also be interpreted in terms of an information state. Linear finite-dimensional recursions involving these recursive estimates are then derived. We also provide some specific choices for such real functions which are motivated by practical applications in robust estimation and filtering etc.

Suppose $m_{k}\left(X_{k}\right)$ is any scalar-valued real function of $X_{k}, k \in \mathbb{N}$. Define $M_{k}=\prod_{l=0}^{k} m_{l}\left(X_{l}\right)$. We wish to obtain a recursion for

$$
E\left[M_{k} \mid \mathcal{Y}_{k}\right]=\frac{\bar{E}\left[\bar{\Lambda}_{k} M_{k} \mid \mathcal{Y}_{k}\right]}{\bar{E}\left[\bar{\Lambda}_{k} \mid \mathcal{Y}_{k}\right]}
$$

Note that the denominator in the right hand side of the previous equation is just a normalizing factor.

Now, define

$$
\begin{aligned}
& B_{k}\left(y_{k}\right)=\operatorname{diag}\left\{\frac{\phi\left[y_{k}-C\left(e_{1}\right)\right]}{\phi\left(y_{k}\right)}, \cdots, \frac{\phi\left[y_{k}-C\left(e_{N}\right)\right]}{\phi\left(y_{k}\right)}\right\} \\
& D_{k}\left(y_{k}\right)=\operatorname{diag}\left\{m_{k}\left(e_{1}\right), \cdots, m_{k}\left(e_{N}\right)\right\}
\end{aligned}
$$

and $\langle a, b\rangle$ as the inner product of two vectors $a$ and $b$.

Definition IV.1: The unnormalized measure $\alpha_{k}$ is

$$
\alpha_{k}=\bar{E}\left[\bar{\Lambda}_{k} M_{k} X_{k} \mid \mathcal{Y}_{k}\right] \text {. }
$$

Lemma IV.1: The unnormalized measure $\alpha_{k}$ obeys the following recursion

$$
\alpha_{k}=B_{k}\left(y_{k}\right) D_{k}\left(y_{k}\right) A \alpha_{k-1} .
$$

Proof:

$$
\begin{aligned}
\alpha_{k}= & \bar{E}\left\{\bar{\Lambda}_{k-1} M_{k-1} \frac{\phi\left[y_{k}-C\left(X_{k}\right)\right]}{\phi\left(y_{k}\right)} m_{k}\left(X_{k}\right) X_{k} \mid \mathcal{Y}_{k}\right\} \\
= & \sum_{i=1}^{N} \bar{E}\left[\bar{\Lambda}_{k-1} M_{k-1}\left\langle X_{k}, e_{i}\right\rangle \mid \mathcal{Y}_{k}\right] e_{i} \\
& \cdot \frac{\phi\left[y_{k}-C\left(e_{i}\right)\right]}{\phi\left(y_{k}\right)} m_{k}\left(e_{i}\right) \\
= & \sum_{i=1}^{N} \frac{\phi\left[y_{k}-C\left(e_{i}\right)\right]}{\phi\left(y_{k}\right)} m_{k}\left(e_{i}\right) \\
& \cdot\left\langle A \bar{E}\left[\bar{\Lambda}_{k-1} M_{k-1} X_{k-1} \mid \mathcal{Y}_{k-1}\right], e_{i}\right\rangle e_{i} \\
= & B_{k}\left(y_{k}\right) D_{k}\left(y_{k}\right) A \alpha_{k-1} .
\end{aligned}
$$

Note IV.1: Note that;

$$
\alpha_{0}=\bar{E}\left[\bar{\Lambda}_{0} M_{0} X_{0} \mid \mathcal{Y}_{0}\right]
$$

and also,

$$
\bar{E}\left[\bar{\Lambda}_{k} M_{k} \mid \mathcal{Y}_{k}\right]=\left\langle\alpha_{k}, \underline{1}\right\rangle
$$

where $\underline{1}=(1,1, \cdots, 1)^{\prime}$

Choices for $M_{k}$ : Now we present two specific choices for $M_{k}$ that have practical applications.

1) Take

$$
m_{k}\left(X_{k}\right)=\left\langle X_{k}, e_{j_{k}}\right\rangle
$$

where $j_{k} \in\{1,2, \cdots, N\}, k \in \mathbb{N}$. In this case, the diagonal matrix $D_{k}, \forall k \in \mathbb{N}$ has only one nonzero entry, namely the $j_{k}$ th term in its diagonal which is 1 . Further,

$\left\langle\alpha_{k}, \underline{1}\right\rangle=\bar{E}\left[\bar{\Lambda}_{k}\left\langle X_{k}, e_{j_{k}}\right\rangle\left\langle X_{k-1}, e_{j_{k-1}}\right\rangle \cdots\left\langle X_{0}, e_{j_{0}}\right\rangle \mid \mathcal{Y}_{k}\right]$.
This is the unnormalized conditional probability that, given $\mathcal{Y}_{k}$, the path of the Markov chain was $e_{j_{0}}, e_{j_{1}}, \cdots, e_{j_{k}}$. In fact, writing $b\left(y_{k}, e_{j_{k}}\right)=\phi\left[y_{k}-C\left(e_{j_{k}}\right)\right] / \phi\left(y_{k}\right)$, we have

$$
\begin{aligned}
\left\langle\alpha_{k}, \underline{1}\right\rangle= & b\left(y_{k}, e_{j_{k}}\right) a_{j_{k} j_{k-1}} b\left(y_{k-1}, e_{j_{k-1}}\right) a_{j_{k-1} j_{k-2}} \\
& \cdots \bar{E}\left[X_{0} \mid \mathcal{Y}_{0}\right] .
\end{aligned}
$$

2) Consider

$$
\begin{aligned}
m_{k}\left(X_{k}, \xi_{k}\right) & =\exp \left\{\theta\left(X_{k}-\xi_{k}\right)^{\prime} Q\left(X_{k}-\xi_{k}\right)\right\} \\
M_{k} & =\prod_{l=0}^{k} m_{l}\left(X_{l}, \xi_{l}\right)
\end{aligned}
$$

where $Q=Q^{\prime}>0$ and $\xi_{k}, k \in \mathbb{N}$ is a sequence of real-valued vectors to be chosen. Note that in this case,

$$
\left\langle\alpha_{k}, \underline{1}\right\rangle=\bar{E}\left[\bar{\Lambda}_{k} M_{k} \mid \mathcal{Y}_{k}\right] .
$$

The parameters $\xi_{0}, \xi_{1}, \cdots, \xi_{k} \in \mathbb{R}^{N}$ are chosen (for $\theta>0$ ) such that $\left\langle\alpha_{k}, \underline{1}\right\rangle$ is minimized. With the choice of $m_{k}\left(X_{k}, \xi_{k}\right)$ given by (9), the problem of optimal estimation of $\xi_{k}, k \in$ IN has been denoted as the risk-sensitive filtering/smoothing problem and solved for general nonlinear signal models in [8] and for hidden Markov models in [9].

Sequential computation of $\xi_{0}, \xi_{1}, \cdots, \xi_{k}$ to yield the optimizing values $\hat{\xi}_{0}, \hat{\xi}_{1}, \cdots, \hat{\xi}_{k}$ relates to the problem of risk-sensitive filtering. But note that they can be chosen at the same time $k$ to yield smoothed estimates. In this brief, we will be concerned about obtaining filtered estimates and not smoothed estimates. Hence, let us consider sequential estimation. Assume that $\hat{\xi}_{0}, \hat{\xi}_{1}, \cdots, \hat{\xi}_{k-1}$ have been chosen. Then $\hat{\xi}_{k} \in \mathbb{R}^{N}$ can be chosen such that

$$
\begin{aligned}
& \hat{\xi}_{k}=\underset{\xi_{k} \in \mathbf{R}^{N}}{\operatorname{argmin}}\left\langle\alpha_{k}\left(\xi_{k}\right), \underline{1}\right\rangle \\
&= \bar{E}\left[\bar{\Lambda}_{k} m_{0}\left(X_{0}, \hat{\xi}_{0}\right)\right. \\
&\left.\cdots m_{k-1}\left(X_{k-1}, \hat{\xi}_{k-1}\right) m_{k}\left(X_{k}, \xi_{k}\right) \mid \mathcal{Y}_{k}\right] .
\end{aligned}
$$

Theorem IV.1: The optimizing value $\hat{\xi}_{k}$ defined previously is given by the solution of the system of equations

$$
\hat{\xi}_{k}=\left[\sum_{j=1}^{N} \rho_{j} m_{k}\left(e_{j}, \hat{\xi}_{k}\right)\right]^{-1}\left[\sum_{j=1}^{N} \rho_{j} m_{k}\left(e_{j}, \hat{\xi}_{k}\right) e_{j}\right]
$$

where

$$
\begin{aligned}
\rho_{j}= & b\left(y_{k}, e_{j}\right) \bar{E}\left[\bar{\Lambda}_{k-1} m_{0}\left(X_{0}, \hat{\xi}_{0}\right)\right. \\
& \left.\cdots m_{k-1}\left(X_{k-1}, \hat{\xi}_{k-1}\right)\left\langle X_{k}, e_{j}\right\rangle \mid \mathcal{Y}_{k}\right] .
\end{aligned}
$$

Proof: Write

$$
\begin{aligned}
\Psi(\xi)= & \bar{E}\left[\bar{\Lambda}_{k} m_{0}\left(X_{0}, \hat{\xi}_{0}\right) \cdots m_{k-1}\left(X_{k-1} \hat{\xi}_{k-1}\right)\right. \\
& \left.\cdots m_{k}\left(X_{k}, \xi\right) \mid \mathcal{Y}_{k}\right] \\
= & \sum_{j=1}^{N} b\left(y_{k}, e_{j}\right) \bar{E}\left[\bar{\Lambda}_{k-1} m_{0}\left(X_{0}, \hat{\xi}_{0}\right)\right. \\
& \left.\cdots m_{k-1}\left(X_{k-1}, \hat{\xi}_{k-1}\right)\left\langle X_{k}, e_{j}\right\rangle \mid \mathcal{Y}_{k}\right] m_{k}\left(e_{j}, \xi\right) \\
= & \sum_{j=1}^{N} \rho_{j} \exp \left\{\theta\left(e_{j}-\xi\right)^{\prime} Q\left(e_{j}-\xi\right)\right\} .
\end{aligned}
$$

Differentiating with respect to $\xi$, we have

$$
\nabla \Psi(\xi)=2 \theta Q \sum_{j=1}^{N}\left(e_{j}-\xi\right) \rho_{j} m_{k}\left(e_{j}, \xi\right) .
$$


As $|\xi| \rightarrow \infty, \Psi(\xi) \rightarrow \infty$. Hence the minimum of $\Psi$ will occur at the critical point when $\nabla \Psi(\xi)=0$, that is when

$$
\sum_{j=1}^{N}\left(e_{j}-\xi\right) \rho_{j} m_{k}\left(e_{j}, \xi\right)=0
$$

Consequently, the critical point is the solution of the system of equations

$$
\xi=\left[\sum_{j=1}^{N} \rho_{j} m_{k}\left(e_{j}, \xi\right)\right]^{-1}\left[\sum_{j=1}^{N} \rho_{j} m_{k}\left(e_{j}, \xi\right) e_{j}\right]
$$

and the proof is complete.

Remark IV.1: Choosing $\xi=\left(\xi_{0}, \xi_{1}, \cdots, \xi_{k}\right)$ at the same time given $\mathcal{Y}_{k}$, gives a robust estimate of the distribution of the whole path to time $k$. In fact, for $1 \leq i \leq N, 0 \leq j \leq k$, write

$$
\begin{aligned}
\rho_{j_{i}}(\xi)= & b\left(y_{i}, e_{j}\right) \bar{E}\left[\bar{\lambda}_{i-1} \bar{\lambda}_{i+1} \cdots \bar{\lambda}_{k} m_{0}\left(X_{0}, \xi_{0}\right)\right. \\
& \cdots m_{i-1}\left(X_{i-1}, \xi_{i-1}\right)\left\langle X_{i}, e_{j}\right\rangle m_{i+1}\left(X_{i+1}, \xi_{i+1}\right) \\
& \left.\cdots m_{k}\left(X_{k}, \xi_{k}\right) \mid \mathcal{Y}_{k}\right] .
\end{aligned}
$$

Then, given $\mathcal{Y}_{k}$, the minimizing values $\left(\hat{\xi}_{0}, \hat{\xi}_{1}, \cdots, \hat{\xi}_{k}\right)$ of $\left(\xi_{0}, \xi_{1}, \cdots, \xi_{k}\right)$ chosen at the same time are the solution of the system equations

$$
\hat{\xi}_{i}=\left[\sum_{j=1}^{N} \rho_{j_{i}}(\hat{\xi}) m_{i}\left(e_{j}, \hat{\xi}_{i}\right)\right]^{-1}\left[\sum_{j=1}^{N} \rho_{j_{i}}(\hat{\xi}) m_{i}\left(e_{j}, \hat{\xi}_{i}\right) e_{j}\right]
$$

\section{VITERBI-TYPE RECURSIONS}

In this section, we use recursions derived in the previous section and show how we can derive well-known recursions like recursions for the "forward variable" in HMM estimation [13] and recursions for Viterbi algorithm for Maximum Likelihood sequence estimations. Then, we present a risk-sensitive version of the Viterbi recursion and show how we can obtain more robust state estimates using this risk-sensitive estimation scheme similar to [8] and [9].

We have seen above from (7) that

$$
\begin{aligned}
& \bar{E}\left[\bar{\Lambda}_{k}\left\langle X_{k}, e_{j_{k}}\right\rangle\left\langle X_{k-1}, e_{j_{k-1}}\right\rangle \cdots\left\langle X_{0}, e_{j_{0}}\right\rangle \mid \mathcal{Y}_{k}\right] \\
& \quad=b\left(y_{k}, e_{j_{k}}\right) a_{j_{k} j_{k-1}} b\left(y_{k-1}, e_{j_{k-1}}\right) a_{j_{k-1} j_{k-2}} \cdots \bar{E}\left[X_{0} \mid \mathcal{Y}_{0}\right]
\end{aligned}
$$

Now, supposing that we sum over all possible values for $e_{j_{k-1}}, e_{j_{k-2}}, \cdots e_{j_{0}}$, we can evaluate $\alpha_{k}\left(j_{k}\right)=$ $\bar{E}\left[\bar{\Lambda}_{k}\left\langle X_{k}, e_{j_{k}}\right\rangle \mid \mathcal{Y}_{k}\right]$ using the fact that $\sum_{j_{k-1}=1}^{N}\left\langle X_{k-1}, e_{j_{k-1}}\right\rangle=1$ etc.

Performing the same summation up to time $k$ for $\alpha_{k+1}\left(j_{k+1}\right)$, we see that

$$
\alpha_{k+1}\left(j_{k+1}\right)=b\left(y_{k+1}, e_{j_{k+1}}\right) \sum_{j_{k}=1}^{N} a_{j_{k+1} j_{k}} \alpha_{k}\left(j_{k}\right) .
$$

This is the usual recurrence for the HMM forward variable, which, in other words is the true filtered estimate of the state given the observations. Similar summations give the recurrence relations for the HMM backward variables, smoothers and transitions (see [12]).

Now consider a similar recurrence relation; but instead of summing over all possible values of the state, let us incorporate a maximization operation. In other words, suppose this recursive variable has been computed up to time $k$ giving a "state" $\left[\delta_{k}(1), \delta_{k}(2), \cdots, \delta_{k}(N)\right]$. Then $\delta_{k+1}(i)$ is defined by

$$
\delta_{k+1}(i)=b\left(y_{k+1}, e_{i}\right) \max _{j} a_{i j} \delta_{k}(j)
$$

This is the usual recurrence relation for the classical Viterbi algorithm for Maximum Likelihood sequence estimation (see [13]). It associates with state $i$ at time $k+1$ a weighted version of the most likely transition to that state.

Now, consider the selection

$$
\begin{aligned}
m_{k}\left(X_{k}, \xi_{k}\right) & =\exp \left\{\theta\left(X_{k}-\xi_{k}\right)^{\prime} Q\left(X_{k}-\xi_{k}\right)\right\} \\
M_{k} & =\prod_{l=0}^{k} m_{l}\left(X_{l}, \xi_{l}\right)
\end{aligned}
$$

Suppose states $\left[\delta_{l}(1), \cdots, \delta_{l}(N)\right]$ have been defined for $l=$ $0,1, \cdots, k$. Let us define the following recursive process with $\delta_{k}$,

$$
\delta_{k+1}(i)=b\left(y_{k+1}, e_{i}\right) m_{k+1}\left(e_{i}, \xi_{k+1}\right) \max _{j} a_{i j} \delta_{k}(j) \text {. }
$$

However, note that $\xi_{k+1}$ is still a free variable. It should be chosen so that it is a good estimate of $X_{k+1}$ given $\mathcal{Y}_{k+1}$.

Consequently, a possible value of $\xi_{k+1}$ would be $E\left[X_{k+1} \mid \mathcal{Y}_{k+1}\right]$ and this could be obtained from a separate HMM filter. Alternatively a robust estimate for $\xi_{k+1}$ could be used, as defined above in Theorem IV.1. However, an estimate obtained from the available parameters would be more satisfactory. Note that if

$$
\hat{\delta}_{k+1}(i)=b\left(y_{k+1}, e_{i}\right) \max _{j} a_{i j} \delta_{k}(j)
$$

then $\hat{\delta}_{k+1} \triangleq\left[\hat{\delta}_{k+1}(1), \cdots, \hat{\delta}_{k+1}(N)\right]$ is approximately an unnormalized conditional distribution for $X_{k+1}$ given $\mathcal{Y}_{k+1}$. Therefore, a surrogate for $E\left[\exp \left\{\theta\left(X_{k+1}-\xi_{k+1}\right)^{\prime} Q\left(X_{k+1}-\xi_{k+1}\right)\right\} \mid \mathcal{Y}_{k+1}\right]$ is given by

$$
\Psi\left(\xi_{k+1}\right)=\sum_{i=1}^{N} \hat{\delta}_{k+1}(i) \exp \left\{\theta\left(e_{i}-\xi_{k+1}\right)^{\prime} Q\left(e_{i}-\xi_{k+1}\right)\right\}
$$

Then, following the same derivation as for Theorem IV.1, the critical values $\hat{\xi}_{k+1}=\left[\hat{\xi}_{k+1}(1), \hat{\xi}_{k+1}(2), \cdots, \hat{\xi}_{k+1}(N)\right]$ for $\Psi\left(\xi_{k+1}\right)$ are the solutions of the system of equations

$$
\hat{\xi}_{k+1}=\frac{\left[\sum_{j=1}^{N} \hat{\delta}_{k+1}(j) m_{k+1}\left(e_{j}, \hat{\xi}_{k+1}\right) e_{j}\right]}{\left[\sum_{j=1}^{N} \hat{\delta}_{k+1}(j) m_{k+1}\left(e_{j}, \hat{\xi}_{k+1}\right)\right]}
$$

Definition V.1: The risk-sensitive Viterbi state is $\delta_{k+1}=$ $\left[\delta_{k+1}(1), \cdots, \delta_{k+1}(N)\right]$ where

$$
\delta_{k+1}(i)=\hat{\delta}_{k+1}(i) m_{k+1}\left(e_{i}, \hat{\xi}_{k+1}\right) .
$$

Remark V.I: Note that as $\theta \rightarrow 0, \delta_{k+1}(i) \rightarrow \hat{\delta}_{k+1}(i), \forall k \in$ $\mathbb{N}$, which implies that RSMLSE approaches the standard Viterbi algorithm.

Remark V.2: Note that a hard decision about the state sequence can be made by storing the arguments $\operatorname{argmax}_{j}\left[\delta_{k}(j) a_{i j}\right], \forall i \in$ $\{1,2, \cdots, N\}, \forall k=0,1, \cdots, T-1$ when a data sequence of fixed length $T$ is available, and then a state sequence backtracking as discussed in [13] for standard Viterbi algorithm for Maximum Likelihood sequence estimation. But, simulation studies show that choosing $\operatorname{argmax}_{j \in\{1,2, \ldots, N\}} \hat{\xi}_{k}(j)$ as the relevant state estimate ensures improved estimation in the presence of parameter uncertainties, particularly, uncertainties in the transition probability matrix $A$.

\section{Continuous-Range State Models}

In this section, we present the risk-sensitive Maximum Likelihood estimation results for continuous-range state models. Consider the probability space $(\Omega, \mathcal{F}, \bar{P})$, under which $\left\{w_{k}\right\}$ and $\left\{y_{k}\right\}$ are sequences of i.i.d random variables with densities $\psi$ and $\phi$, respectively. 
Suppose the state variable evolves according to the following dynamics

$$
x_{k+1}=f\left(x_{k}\right)+w_{k+1}
$$

where $x_{k} \in \mathbb{R}$ is scalar for simplicity.

Remark VI.1: All our results are easily extendible to a vector state process although we will consider a scalar state process here for simplicity.

Define

$$
\begin{aligned}
& \bar{\lambda}_{k}=\frac{\phi\left[y_{k}-c\left(x_{k}\right)\right]}{\phi\left(y_{k}\right)}, \\
& \bar{\Lambda}_{k}=\prod_{l=0}^{k} \bar{\lambda}_{l}
\end{aligned}
$$

where

$$
v_{k}=y_{k}-c\left(x_{k}\right)
$$

Also define $\mathcal{G}_{k}^{0}=\sigma\left\{X_{0}, X_{1}, \cdots, X_{k}, y_{0}, y_{1}, \cdots, y_{k-1}\right\}$ and $\mathcal{Y}_{k}^{0}=\sigma\left\{y_{0}, y_{1}, \cdots, y_{k}\right\}$ and the corresponding complete filtrations as $\left\{\mathcal{G}_{k}\right\}$ and $\left\{\mathcal{Y}_{k}\right\}$. Using the change of probability measure techniques discussed in Section III, we define a measure $P$ by setting $d P /\left.d \bar{P}\right|_{\mathcal{G}_{k}}=\bar{\Lambda}_{k}$. Then (see [12]) under $P, v_{k}, k \in \mathbb{N}$ are i.i.d with densities $\phi$.

Let $g$ be any bounded, measurable test function and suppose $q_{k}($. is the unnormalized conditional density so that

$$
\bar{E}\left[\bar{\Lambda}_{k} g\left(x_{k}\right) \mid \mathcal{Y}_{k}\right]=\int_{\mathbf{R}} g(x) q_{k}(x) d x
$$

Then it is shown in [12] that $q_{k}$ satisfies the recurrence relation

$$
q_{k+1}(x)=\frac{\phi\left[y_{k+1}-c(x)\right]}{\phi\left(y_{k+1}\right)} \int_{\mathbf{R}} \psi[x-f(z)] q_{k}(z) d z .
$$

Replacing the integration by a maximization, we have the following recursion for a continuous-range state Maximum Likelihood estimation algorithm.

Suppose $\hat{\delta}_{l}(x)$ has been defined for $0 \leq i \leq k$. Then, the relevant recursion in $\hat{\delta}_{k}(x)$ is given by

$$
\hat{\delta}_{k+1}(x)=\frac{\phi\left[y_{k+1}-c(x)\right]}{\phi\left(y_{k+1}\right)} \sup _{z} \psi[x-f(z)] \hat{\delta}_{k}(z) .
$$

Define $\Psi(\xi)=\int_{\mathbf{R}} \hat{\delta}_{k+1}(x) \exp \left\{\theta(x-\xi)^{\prime} Q(x-\xi)\right\} d x$. Then $\nabla \Psi(\xi)=0$, when

$$
\xi=\frac{\int_{\mathbf{R}} x \hat{\delta}_{k+1}(x) \exp \left\{\theta(x-\xi)^{\prime} Q(x-\xi)\right\} d x}{\int_{\mathbf{R}} \hat{\delta}_{k+1}(x) \exp \left\{\theta(x-\xi)^{\prime} Q(x-\xi)\right\} d x} .
$$

Writing $\hat{\xi}_{k+1}$ for the solution of (25), we have the risk-sensitive Maximum Likelihood estimate defined as

$$
\delta_{k+1}(x)=\exp \left\{\theta\left(x-\hat{\xi}_{k+1}\right)^{\prime} Q\left(x-\hat{\xi}_{k+1}\right)\right\} \hat{\delta}_{k+1}(x)
$$

Remark VI.2: Note that when the dynamics are linear and the noise is Gaussian, the recursive Maximum Likelihood estimate $\delta_{k+1}(x)$ is the same as that given by the Kalman filter.
TABLE I

Performance Comparison of RSMLSE and Standard Viterbi Algorithms

\begin{tabular}{l|c|c}
\multicolumn{2}{c}{$\sigma=0.5, \theta=2.0$} \\
\hline \multirow{2}{*}{ Algorithm } & \multicolumn{2}{|c}{ No: of decision errors } \\
\cline { 2 - 3 } & $a_{11}=0.1 a_{21}=0.9$ & $a_{11}=0.3 a_{21}=0.7$ \\
\hline Viterbi & 1279 & 464 \\
\hline RSMLSE & 560 & 338 \\
\hline \multicolumn{3}{|c}{$\sigma=1.0, \theta=2.0$} \\
\hline \multirow{2}{*}{ Algorithm } & \multicolumn{2}{|c}{ No: of decision errors } \\
\cline { 2 - 3 } & $a_{11}=0.1 a_{21}=0.9$ & $a_{11}=0.3 a_{21}=0.7$ \\
\hline Viterbi & 4136 & 2851 \\
\hline RSMLSE & 2668 & 2110 \\
\hline
\end{tabular}

\begin{tabular}{l|c|c}
\multicolumn{3}{|c}{$\sigma=2.0, \theta=2.0$} \\
\hline \multirow{2}{*}{ Algorithm } & \multicolumn{2}{|c}{ No: of decision errors } \\
\cline { 2 - 3 } & $a_{11}=0.3 a_{21}=0.7$ & $a_{11}=0.4 a_{21}=0.6$ \\
\hline Viterbi & 4444 & 3925 \\
\hline RSMLSE & 3692 & 3468 \\
\hline
\end{tabular}

\section{Simulation StUdiES}

In this section, simulation results are presented regarding the robustness of the risk-sensitive Maximum Likelihood estimation algorithm over the standard Viterbi algorithm for Markovian input data. It is seen that the standard Viterbi algorithm is quite sensitive to uncertainties in the transition probabilities, particularly if the transition probability matrix being used by the standard Viterbi algorithm is quite different to the actual one. In such cases, the robust (risk-sensitive) algorithm gives less number of errors in the recovered data. The details of the simulations are given below.

Simulations have been carried out with a 2-state Markov chain $X_{k}$ generated with transition probability values $a_{11}=0.9, a_{12}=0.1$.

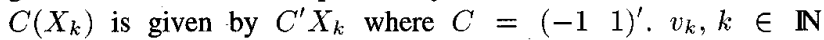
is distributed with $N\left(0, \sigma^{2}\right)$. They are based on a set of 10000 data points. Table I shows the improvement achieved by the robust algorithm over the standard Viterbi algorithm in terms of number of errors obtained in recovered data, for different incorrect transition probability values and different noise variances. Simulations with other sets of random data have been done and the results obtained are consistent.

Remark VII.1: In cases where the transition probability matrix used by the algorithms is close to the actual one, the robust algorithm does not give a substantial improvement over the standard Viterbi algorithm. Simulations have been carried out for other sorts of uncertainties, particularly in the noise statistics. In these situations the risk-sensitive algorithm does not perform any better than the standard Viterbi algorithm. But it has been noted in [9], risksensitive state estimation for HMM's gives better performance than standard minimum variance estimation under uncertainties in the noise statistics. Hence, the authors suggest that in situations where there are uncertainties in the transition probability matrix and the noise statistics, a hybrid algorithm consisting of risk-sensitive filtering and risk-sensitive Maximum Likelihood estimation combined in an effective (of course suboptimal) way can be applied. Details are still under investigation.

Remark VII.2: It is also worth pointing out that applications of risk-sensitive algorithms are slowly emerging in literature such 
as fault detection [16]. The authors' experience says that such applications are very specific and case-sensitive. Hence, the risksensitive algorithm proposed in this paper is not expected to perform better than the standard Viterbi algorithm under all sorts of uncertain situations. Of course, further research in this direction is necessary.

\section{CONCLUSION}

In conclusion, it will be fair to say that the proposed risk-sensitive Maximum Likelihood sequence estimator achieves the robustness expected from risk-sensitive algorithms in the case of uncertainty in the transition probability matrix of the Markov chain. More research is still needed to understand the behavior of risk-sensitive algorithms with changing values of $\theta$, the risk-sensitive parameter. Also, using the techniques of [10], RSMLSE can be interpreted in terms of an estimation problem in a deterministic worst-case noise scenario. Of course, RSMLSE becomes the standard Viterbi algorithm as $\theta \rightarrow 0$.

\section{REFERENCES}

[1] P. Whittle, "Risk-sensitive linear/quadratic/Gaussian control," $A d v$. Applied Probability 13, App. Prob. Trust, pp. 746-777, 1981.

[2] _ "A risk-sensitive maximum principle: The case of imperfect state observation;" IEEE Trans. Automat. Contr., vol. 36, pp. 793-801, 1991.

[3] A. Bensoussan and J. H. van Schuppen, "Optimal control of partially observable stochastic systems with an exponential-of-integral performance index.," SIAM J. Contr. Opt., vol. 23, pp. 599-613, 1985.

[4] D. H. Jacobson, "Optimal stochastic linear systems with exponential performance criteria and their relation to deterministic games," IEEE Trans. Automatic Contr., vol. AC-18, pp. 124-131, Apr. 1973.
[5] I. B. Collings, M. R. James, and J. B. Moore, "An information-state approach to risk-sensitive tracking problems," submitted to J. Math. Syst., Est., Contr., 1994.

[6] J. L. Speyer, C. Fan, and R. N. Banavar, "Optimal stochastic estimation with exponential cost criteria," in Proc. 31 st Conf. Decision Contr., Dec. 1992, vol. 2, pp. 2293-2298.

[7] M. R. James, J. S. Baras, and R. J. Elliott, "Risk-sensitive control and dynamic games for partially observed discrete-time systems," IEEE Trans. Automat. Contr., vol. 39, pp. 780-792, Apr. 1994.

[8] S. Dey and J. B. Moore, "Risk-sensitive filtering and smoothing via reference probability methods," in Proc. Amer. Contr. Conf., Seattle, WA, June 1995, vol. 1, pp. 129-133.

[9] _ _ "Risk-sensitive filtering and smoothing for hidden Markov models," Syst. Contr. Lett., vol. 25, no. 5, pp. 361-366, Aug. 1995.

[10] J. B. Moore, R. J. Elliott, and S. Dey, "Risk-sensitive generalizations of minimum variance estimation and control," J. Math. Syst., Est. Contr., accepted for publication, 1995.

[11] R. J. Elliott and H. Yang, "Forward and backward equations for an adjoint process," Festchrift for G. Kallianpur. Berlin: New York: Heidelberg, Springer Verlag, pp. 61-70.

[12] R. J. Elliott, L. Aggoun, and J. B. Moore, Hidden Markov Models: Estimation and Control. New York: Springer-Verlag, 1994.

[13] L. R. Rabiner, "A tutorial on hidden Markov models and selected applications in speech recognition," Proc. IEEE, vol. 77, pp. 257-285, 1989.

[14] G. D. Forney, "The Viterbi algorithm," Proc. IEEE, vol. 61, pp. 268-278, Mar. 1973.

[15] A. Segall, "Recursive estimation from discrete-time point processes," IEEE Trans. Inform. Theory, vol. IT-22, pp. 422-431, 1976.

[16] R. Mangoubi, "Robust and risk-sensitive estimation with application to failure detection and isolation," Ph.D. disssertation, Dept. Aeronaut. Astronaut., MTT. 